\title{
Design of Greenhouse Control Engine of Optimal to Minimize the Energy Costs
}

\author{
Miran Baek, MyeongBae Lee, Jangwoo Park, Yongyun Cho and Changsun Shin* \\ Dept. Information and Communication Engineering, Sunchon National University, \\ 255 Jungangno, Suncheon, Jeonnam 540-950, Republic of Korea \\ \{tm904,lmb, jwpark,yycho,csshin\}@sunchon.ac.kr
}

\begin{abstract}
In this paper, I have revised and supplement the Greenhouse Control System (GCS) proposed by existing. In addition, we studied the detailed design of the Greenhouse Control Engine (GCE) which is one of the engine that make up the GCS. GCE was designed to place the purpose of minimizing the cost consumed by the control device in the operation for the optimal control of the crop growth environment. The GCE consist of Data Aggregator (DA) to collect information of the greenhouse, Greenhouse Information Storage (GIS) stores the collected information, and Greenhouse Control Agent (GCA) is based on analysis of stored data and considering energy-efficient select the appropriate control devices. Also, this engine provides the greenhouse environment information collection and analysis service and the control device select service. And tested the designed GCE through the test bed.
\end{abstract}

Keywords: Greenhouse Control Engine, u-IT, Greenhouse Cultivation, Energy Costs, Automatic Control

\section{Introduction}

Recently, farm village are being introduced new advanced cultivation system such as smart greenhouse or smart farm through the fusion of u-IT technology. Thus, the reason for introducing the u-IT technology of agriculture there is a variety of reasons such as ease of cultivation, increase in production, Labor savings [1]. However, the most important reason the farms will affect the farms total income by applying u-IT technology. Total income of farms can be calculated by the difference of the cost consumed during the crop cultivation period and a total sales income of agricultural products. Thus, when they are based on calculations of total income, no matter how good the margin is, even if the cost of energy consumed to control the growth environment of crop, many the farms income will remain lower. Thus, by factors that increase the income of famers to grow crops is also important to reduce the energy costs.

In this paper, in order to optimize the consumption cost with the optimal growth environment, GCE is used which was also suggested previously in the GCS [6], a study of detailed design.

This paper is organized as follows. Chapter 2 describes about the related work. Chapter 3 details the functional design of the GCE and test of the GCE. Finally, Chapter 4 consists of conclusion.

${ }^{*}$ Corresponding Author 


\section{Related Work}

Recently, agriculture is u-IT technology combined smart agriculture is being realized. At the core of u-IT technology can be used as a technology that invisible computing environment of the user's anytime, anywhere, by anyone, in any network, any device [2]. Utilizing the u-IT technology is many studies have been proceed in domestic and foreign.

HortiMax Company [3, 4] of Nederland to predict weather information facility using weather information and a variety of sensors. And it provides a solution to optimize the temperature deviation in the facility. Also, supports to collect information on the surrounding environment of crop using fruit artificial sensors, infrared temperature sensors, CO2 sensor. It is possible to specify various a control options through the userconfigurable software-based control systems. And, supports to be able to control the same condition the numerous blocks.

ETRI in Korea are recognizing the changes in the environment and growth state by applying the RFID / USN technology, and to control automatically the optimal growth environment. Supports the growth environment and storage environment integrated control for to secure the reliability about the products of consumer [5].

It also monitors environment around of the crop and all acts of a series related to the harvesting of agricultural products through a sensor that measures each temperature, humidity and soil. And, we studied the system to be able to check the harvest at a particular location by measuring the weight of the harvested agricultural and transmits the information to the user's terminal [1].

\section{Greenhouse Control Engine (GCE)}

\subsection{Design of Greenhouse Control Engine}

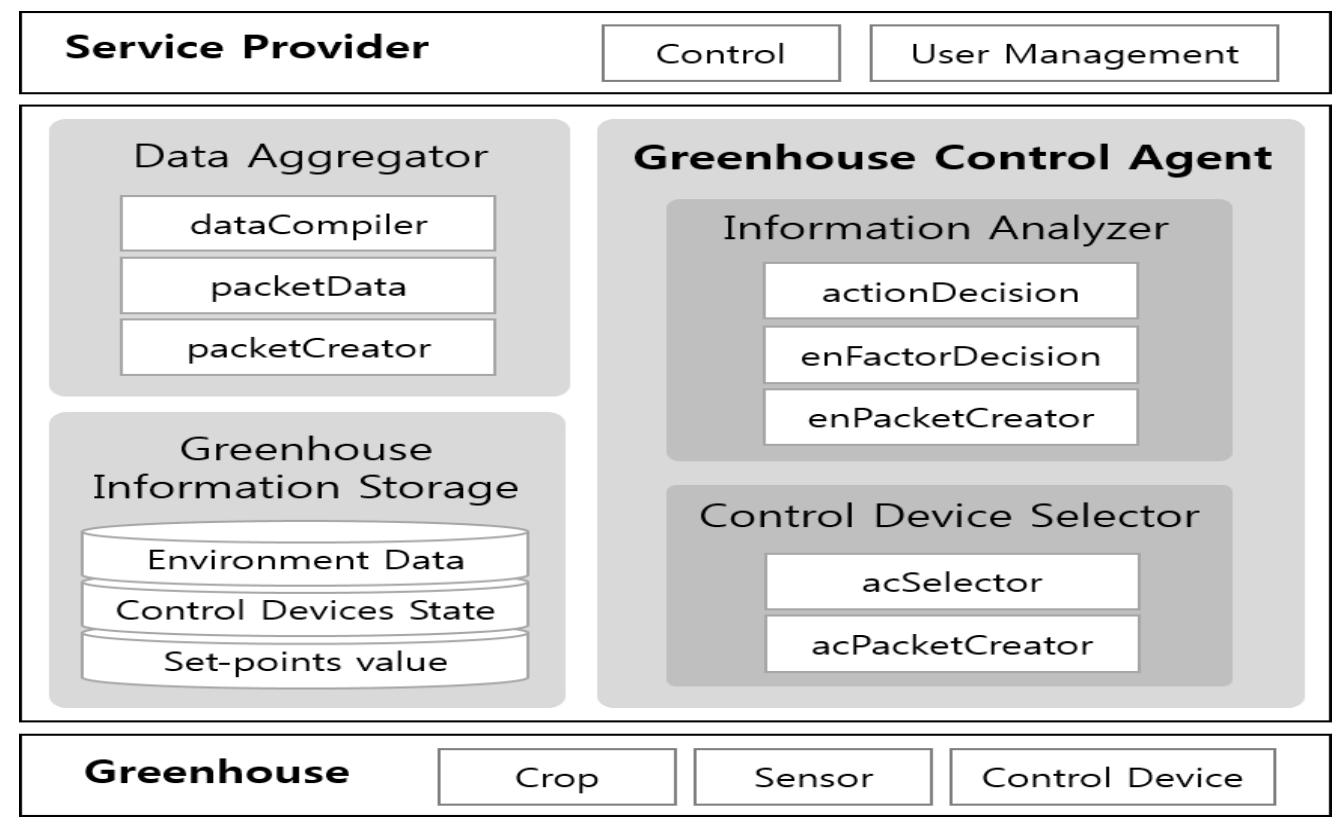

Figure 1. Component of GCE 
In this study, designed GCE is one of the two engines that make up the GCS. The main function is to collect environment data from the greenhouse. And considering the energy-efficiency for create the greenhouse environment of optimal based on the growth environment set-points that are calculated from the Crop Growth Agent (CGA) of the CGE, and carry out the control device selection and device control function. Figure 1 shows the components of the GCE. As the Figure 1. GCE consist of the Data Aggregator (DA) to collect data, the Greenhouse Information Storage (GIS) to store the data, and the Greenhouse Control Agent (GCA) is important function in the GCE. The GCA includes the Information Analyzer (IA) to analyze information and the Control Device Selector (CDS) to select control device.

The functions of each component of GCE are as follows:

- Data Aggregator (DA)

Table 1. The data defined in the packet

\begin{tabular}{cl}
\hline Variable & \multicolumn{1}{c}{ Description } \\
\hline id & id of sensors \\
sensorGroupID & id of sensors group \\
dataCode & type of data to collect from sensors \\
currentData & current collected data from sensor \\
sensorGroupAce & average of currentData of sensors group \\
\hline
\end{tabular}

To collect inside, outside and soil environment information through the inside/outside meteorological sensors and soil sensors. Also, by collecting status information of the current control devices on the GIS is the ability to save.

The DA consists of dataCompiler, packetData, and PacketCreator class. The dataCompiler class is performed the function of converting the data actually availa-ble on the data collected from the sensor. The packetData is a class that defines the packet by adding required information. Table 1 is the data defined in the packet. The packetCreator provides the function to enter data.

\section{- Greenhouse Information Storage (GIS)}

Table 2. The data to be stored in the GIS

\begin{tabular}{cl}
\hline Variable & \multicolumn{1}{c}{ Collect data } \\
\hline Environment Data & Temperature, Humidity, $\mathrm{CO}_{2}$ concentration, Solar radiation \\
Control device Status & $\begin{array}{l}\text { Cooler, Heater, Artificial light, } \mathrm{CO}_{2} \text { generator, Sprinkler, Sky/side } \\
\text { window, Canopy }\end{array}$ \\
Set-points value & Set-points \\
\hline
\end{tabular}


The Greenhouse Information Storage as a storage to store the information of greenhouse, store the greenhouse environment data collected from the DA and the environment set-points that determined from the GCA. Also, store controlled environment data through the GCA and the function to save status of the control devices. Table 2 is the data to be stored in the Greenhouse Information Storage.

\section{- Greenhouse Control Agent (GCA)}

IA is called the data collected from the DA and the set-points that are stored in the storage. And I serves to determine the environment factors to be controlled based on the set-points which is currently determined through algorithm.

The IA consists of actionDecision, enFactorDecision, and enPacketCreator class. The actionDecision is a class that large differences than the error range by comparing previous data and the processed data received from the DA, and to determine whether the analyzer works compared with set-points. The enFactorDecision is a class that to determine the environment factors the final control by analyzing a setData and the setpoints, which is determined by the Environment Set-points Decisioner of the CGA. The enPacketCreator provides data settings and input function. CDS is serves to determine the control value and select the appropriate control devices considering energy-efficient for optimal control determined environment factors from the IA.

The CDS consists of acSelector and acPacketCreator class. The acSelector is a class that control device select and determine the control value by applying the control model that determined environment factors. The acPacketCreator provides data input function.

\subsection{Greenhouse Control Engine Service}

\subsubsection{Greenhouse Environment Information Collection and Analysis Service}

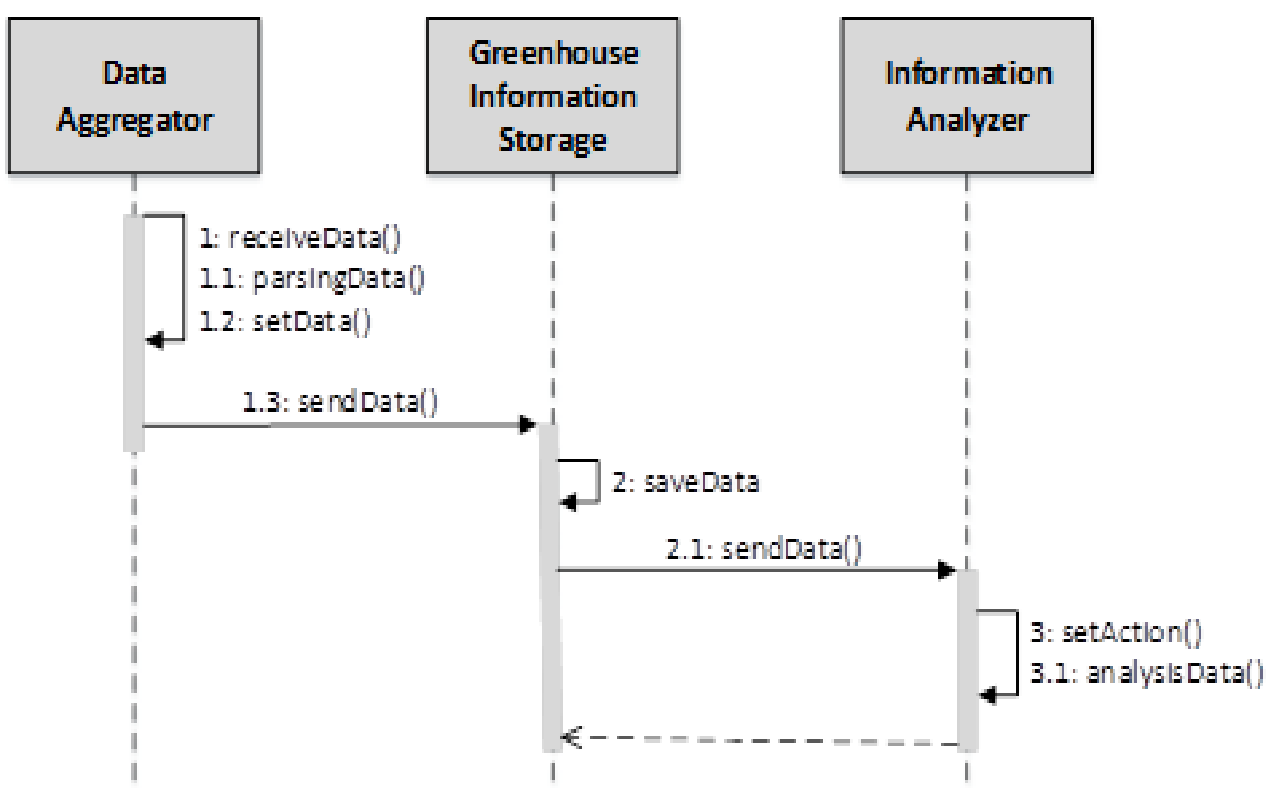

Figure 2. Motion process of Greenhouse Environment Information Collection and Analysis Service 
The greenhouse environment information collection and analysis service is a service that collect environment information and analyze the data in order to optimize the control of greenhouse environment based on the collected data. When a request is made for the data from the DA, the sensed data is transferred through the wireless sensor network. DA generates a data packet and stores the data in the GIS. IA needs the required data from the GIS. The environment factor are determined, once the analyzed data is transferred to the control of greenhouse. The analyzed data are stored in the GIS. The motion process of service is shown in Figure 2.

\subsubsection{Control Device Service}

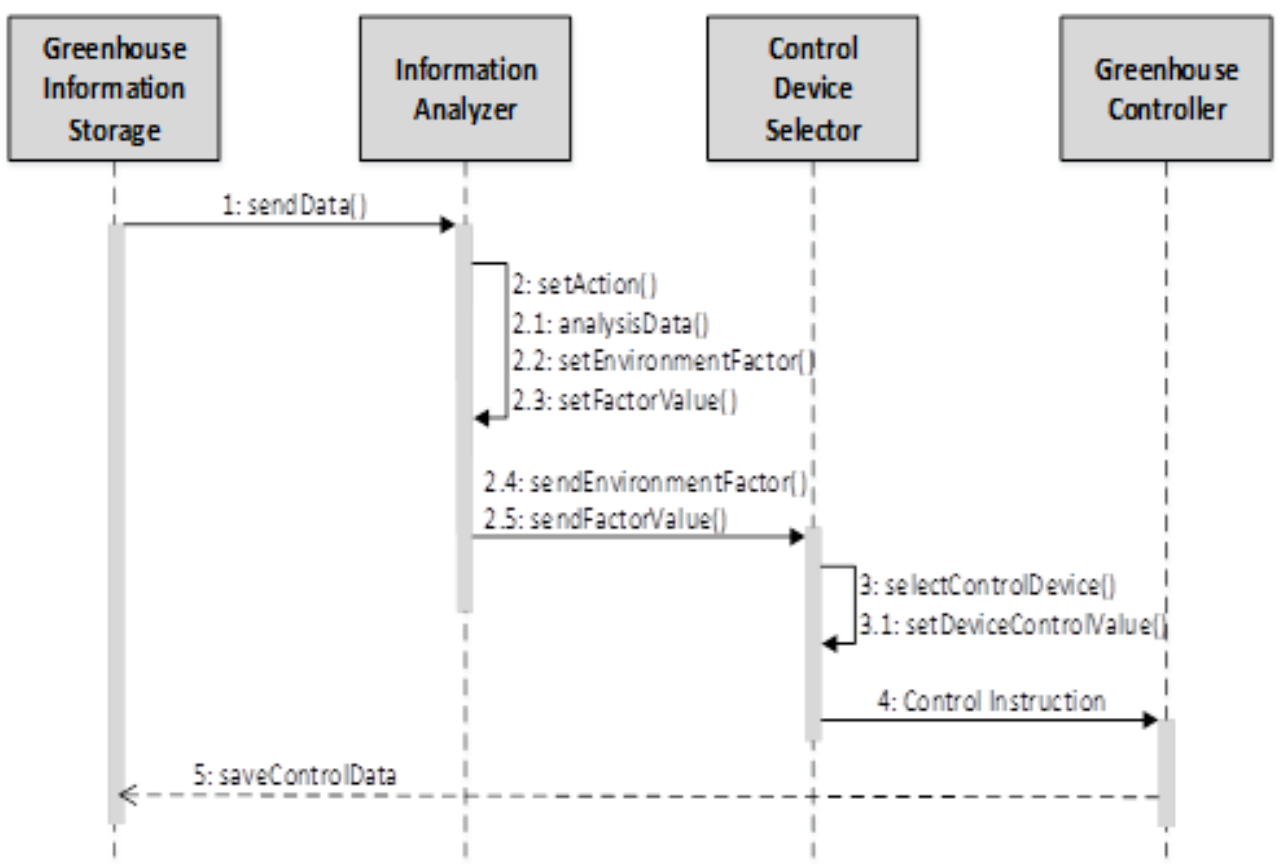

Figure 3. Motion process of Control Device Select Service

The control device select service is a service that select control devices by considering the energy-efficient to control the factors when calculated values of environment factors to control the greenhouse through the IA. Environment data stored in the GIS and set-points are passed to the IA. The IA determines the factors that must be controlled in the greenhouse to analyze the received data and analyzed data is passed to the CDS. CDS selects the control device based on the analysis data that was provided from the IA. It would be considered energy-efficient when selecting the control device. To control certain environment factors, before entering the control devices, it is necessary to consider the energy efficient and low cost. To achieve this, a suitable selection algorithm is performed to select the control devices. The selected control device is stored in the GIS and then sends the control commands to the Greenhouse Controller. The motion process of service is shown in Figure 3.

\subsection{Test of GCE}

As shown in Figure 4 made the test bed to test the designed GCE. Made a virtual environment to collect data using a sensor installed. And the collected data shows in 
real-time. Further, the control device can be seen to work by considering the energy efficiency in order to control the greenhouse environment to suit set-points.

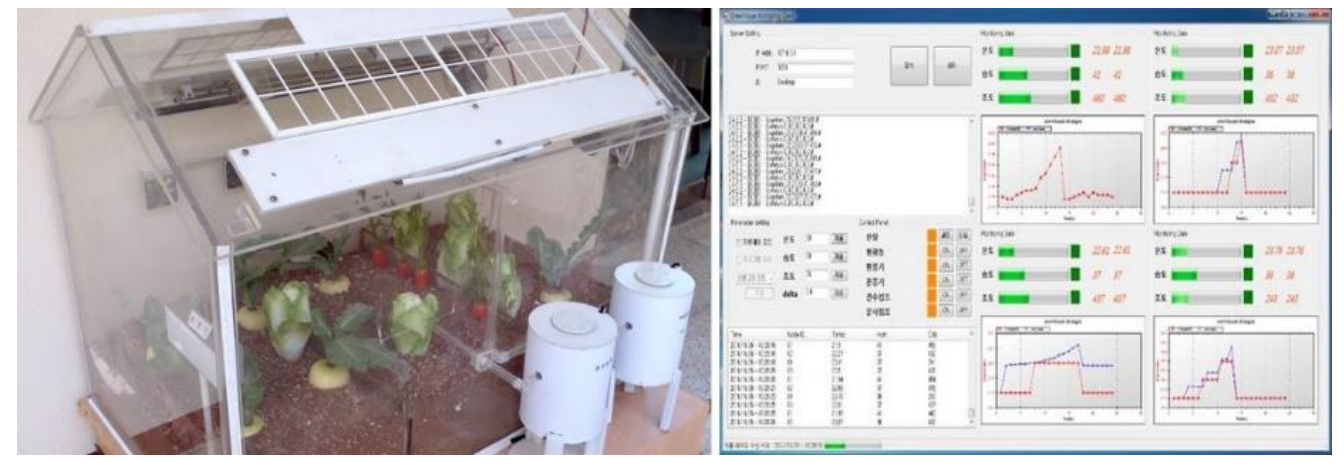

Figure 4. GCE test bed and service applications

\section{Conclusion}

In this paper, in order to optimal control of greenhouse environment control operated the Greenhouse Control Engine (GCE) was about the detailed design. GCE was designed that not only increase in yield is one of the factors to increase the income of farmers and aim to minimize the cost of consumption of the control devices driven for control. This engine consists of Data Aggregator (DA), Greenhouse Information Storage (GIS), and Greenhouse Control Agent (GCA). The DA collects the information of greenhouse, GIS stores the collected information and GCA selects the appropriate control devices based on the analysis of stored data and considering the energyefficiency. Also, this engine provides two services. The greenhouse environment information collection and analysis service and the control device select service. Developed a control model of greenhouse based on the engine designed and we will define the optimal control points. Also, future works we will experiment through a test bed in order to minimize the cost that consumption is driven by the control devices through the development of control selection algorithm.

\section{Acknowledgements}

This research was supported by Basic Science Research Program through the National Research Foundation of Korea (NRF) funded by the Ministry of Education, Science and Technology (20110014742). And this work was supported by the Industrial Strategic technology development program, 10040125, Development of the Integrated Environment Control S/W Platform for Constructing an Urbanized Vertical Farm funded by the Ministry of Knowledge Economy (MKE, Korea).

\section{References}

[1] J. Huidong, H. J. Hwan and H. Yoe, “A Design of Yield Monitoring System for Precision Farming based on u-IT", The Korean Institute of Communications and Information Sciences, no. 6, (2008), pp. 200-203.

[2] J. H. Kim, “(IT) Agriculture IT-Information Technology is Leading the Future”, Rural and Environment, vol. 116, (2012), pp. 14-25.

[3] S. S. Kang, S. H. Kim, J. W. Lee and H. J. Kang, "USN Based Agricultural IT Convergence Technology Trends", Electronics and Telecommunications Trends, vol. 26, no. 6, (2011), pp.97-107.

[4] http://www.horimax.com. 
[5] S. Kyo-Hun, "Greenhouse Environment Wireless Monitoring and Control System", Electronics and Telecommunications Research Institute, (2012), pp.1-10.

[6] M. Baek, M. Lee, H. Kim, T. Kim, N. Bae, Y. Cho, J. Park and C. Shin, "A Novel Model for Greenhouse Control Architecture", Lecture Notes in Computer Science, vol. 7861, (2013), pp.262-269.

[7] Farming daily record, http://blog.daum.net/intel8120/2083325.

[8] F. Rodriguez, M. Berenguel and M. R. Arahal, "A Hierarchical Control System for Maximizing Profit in Greenhouse Crop Production.

\section{Authors}

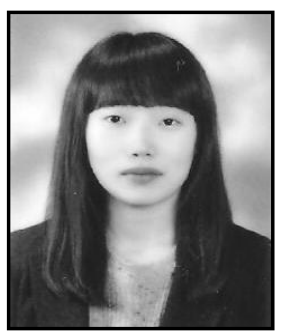

\section{Miran Baek}

She is a Master's course student of Sunchon University. She is also a researcher of Go-Lab at Sunchon National University too. Her main research interests include Ubiquitous Agriculture and Ubiquitous Computing.

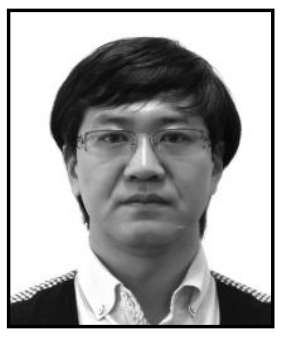

\section{MyeongBae Lee}

MyeongBae Lee is a PhD student of Sunchon National University. He is also a researcher of Go-Lab at Sunchon National University too. His main research interests include Ubiquitous Computing and Middleware.

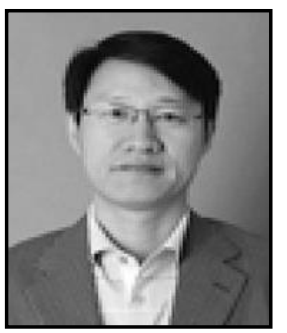

\section{Jangwoo Park}

He received the $\mathrm{BS}, \mathrm{MS}$ and $\mathrm{PhD}$ degrees in Electronic engineering from Hanyang University, Seoul, Korea in 1987, 1989 and 1993, respectively. In 1995, he joined the faculty member of the Sunchon National University, where he is currently a professor in the Department of Information \& Communication engineering. His research focuses on Localization and SoC and system designs and RFID/USN technologies

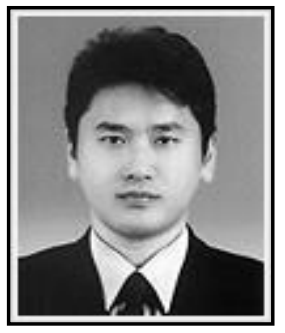

\section{Yongyun Cho}

Yongyun Cho received the $\mathrm{PhD}$ degree in computer engineering at Soongsil University. Currently, he is an assistant professor of the Department of information \& communication engineering in Sunchon National University. His main research interests include System Software, Embedded Software and Ubiquitous Computing. 
International Journal of Control and Automation

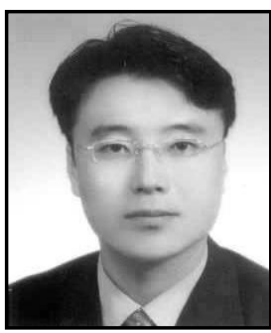

\section{Changsun Shin}

Changsun Shin received the $\mathrm{PhD}$ degree in computer engineering at Wonkwang University. Currently, he is an associate professor of the Department of information \& communication engineering in Sunchon National University. His main research interests include Distributed Real-Time Computing, Distributed Object Modeling, Ubiquitous Agriculture and Ubiquitous Sensor Network (USN). 